\title{
ARTIGOS
}

\section{A PESQUISA EM DESIGN PARA O COMPORTAMENTO SUSTENTÁVEL \\ LACUNAS E DESAFIOS}

\section{Marco Ogê Muniz, MSc (Univille); Aguinaldo dos Santos, Ph.D (UFPR)}

PALAVRAS CHAVE

Design para o Comportamento Sustentável, sustentabilidade, Design Sustentável, comportamento e comportamento do usuário.

\section{KEY WORDS}

Design for Sustainable behavior, sustainability, sustainable design, behavior and user behavior.

\section{RESUMO}

O presente artigo promove a discussão acerca dos desafios da pesquisa em Design para o comportamento sustentável - Design for Sustainable Behavior (DfSB). É apresentada uma breve discussão sobre a definição do termo, sobre comportamentos e hábitos e sobre o projeto da mudança de comportamento. São mostrados exemplos de produtos que proporcionam a mudança de comportamento e as lacunas para futuras pesquisas. Como conclusão é apresentado um panorama geral da pesquisa em DfSB e são mostradas propostas de abordagens para futuras investigações.

\section{ABSTRACT}

This paper promotes discussion of the research challenges in Design for Sustainable Behavior (DfSB). Presented a brief discussion on the definition of the term, on behavior and habits and behavior change project. They appear examples of products that deliver behavior change and gaps for future research. In conclusion we present a overview of research in DfSB approaches and proposals for future research. 


\section{INTRODUÇÃO}

A investigação acerca da temática Design para o Comportamento Sustentável - Design for Sustainable Behaviour (DfSB) - vem se consolidando na pesquisa em Design há cerca de 10 anos. As abordagens recorrentes em DfSB se concentram no estudo dos modelos mentais dos usuários, na aplicabilidade do DfSB, na compreensão do comportamento dos usuários e na tradução das percepções para uso em fases iniciais de projeto e nas intervenções de DfSB e avaliação da sua eficácia (WEVER, 2012). A produção científica corrente sobre DfSB basicamente apresenta, explica e estrutura possíveis estratégias; propõe soluções de design baseadas nessas estratégias e mostra estudos de caso para avaliar a aceitação e a efetividade desses projetos (BOKS, 2012).

O Núcleo de Design e Sustentabilidade (NDS) da Universidade Federal do Paraná (UFPR) promove a investigação em DfSB por meio de projetos de pesquisa. Esses projetos contam com a participação de pesquisadores, dentre os quais doutorando e mestrandos do Programa de Pós-Graduação em Design (PPGDesign) da UFPR, bolsistas de iniciação científica da graduação em Design da instituição. O projeto E-Wise, no ano de 2012, identificou os hábitos de consumo de água e energia nas habitações de interesse social para entender a percepção que as pessoas de baixa renda têm de fontes alternativas de água e energia (E-WISE, 2014); e o projeto Skoon, no ano de 2013, identificou e caracterizou os hábitos e comportamentos dos usuários de habitações de interesse social no uso de máquinas de lavar roupa (SKOON, 2014).

De modo a ampliar a pesquisa sobre o tema, o presente artigo apresenta uma breve discussão sobre o Design para o Comportamento Sustentável (Design for Sustainable Behavior - DfsB) para compreensão do estado da arte da pesquisa acerca do tema. A motivação para a execução desse levantamento bibliográfico surgiu da necessidade de aprofundamento na literatura existente sobre o Design para o Comportamento Sustentável para fundamentação da tese de doutorado do autor. São mostrados os variados campos de aplicação e as diferentes abordagens do tema na pesquisa em design, ao longo dos anos.

A revisão resultou no esclarecimento das pesquisas já realizadas em Design para o Comportamento Sustentável. Este esclarecimento, além de apresentar o avanço da pesquisa em DfSB, esclarece os campos de aplicação, as abordagens de DfSB na pesquisa em design e as lacunas para futuras investigações.

\section{DESIGN PARA O COMPORTAMENTO SUSTENTÁVEL}

Apesar dos seus aspectos terem sido abordados anteriormente na literatura existente, a junção específica dos três aspectos (design, sustentabilidade e comportamento) combinando os pesquisadores com experiência em pesquisa e na prática em design, é relativamente nova (WEVER, 2012). Projetar para a mudança de comportamento integra a pesquisa comportamental, o desenvolvimento pragmático do produto e uma rigorosa análise de dados (WENDEL, 2014).

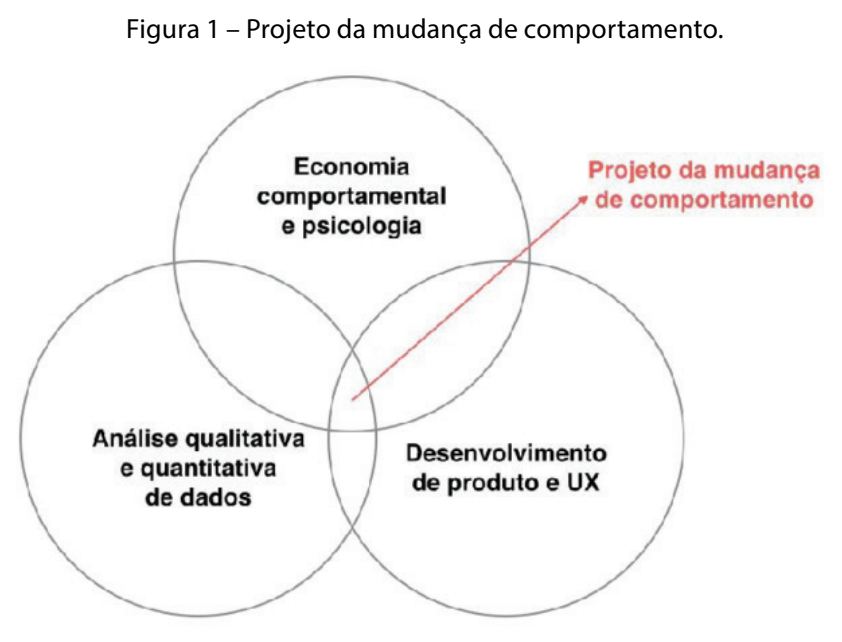

Fonte: Adaptado de Wendel, 2014.

Os campos de aplicação em DfSB são diversos, dentre as quais, em processos de lavagem de roupa (MCCALLEY e MIDDEN, 2002, 2006, 2006a; FROEHLICH, 2009; LAITALA e BOKS, 2012; DAROS, 2013; FORCATO; 2014). Também aparece no uso de energia doméstica em diferentes atividades (RODRÍGUEZ, 2004; RODRÍGUEZ e BOKS, 2005), tais como, em eletrodomésticos (ELIAS, 2007; 2008; ELIAS e CULLEY, 2009), em medidor de energia (WEVER, et. al., 2008) e em telefones celulares e refrigeradores domésticos (LILLEY, 2009; BHAMRA, et. al., 2011), por sistemas de gestão de energia doméstica - Home Energy Management Systems (HEMS) - (VAN DAM, et. al., 2012), pela gamificação (GEELEN, et. al., 2012), para o aquecimento (KUIJER e DE JONG, 2012), em ar condicionado (BHAMRA e TANG, 2012) e pela renovação doméstica do uso de energia (HAINES, V., et. al., 2012). O uso de água é apresentado tanto no banheiro (SCOTT, et. al., 2011) quanto na lavanderia (JELSMA e KNOT, 2002; DAROS, 2013). Por fim, são mostrados o descarte e o uso do lixo (WEVER et. al., 2006; WEVER, et. al., 2011). 


\subsection{Comportamentos e Hábitos}

Kahneman (2012) assegura que o cérebro humano possui basicamente dois modelos de pensamento: o deliberativo e o intuitivo. O modo intuitivo, também chamado de emocional ou sistema 1, funciona de maneira rápida e automática. Ele atua sem a plena consciência do seu funcionamento com base em experiências passadas e um conjunto de regras para dar uma resposta intuitiva. Já o modo deliberativo, também chamado de consciência ou sistema 2, funciona lentamente, de forma focada e consciente. Ele é responsável por lidar com problemas complexos que exigem raciocínio lógico.

A formação de um hábito, em nível cerebral, decorre da variação entre os pensamentos intuitivos e deliberativos. Para Duhigg (2012) o processo de formação de um hábito acontece como um loop de três estágios. Primeiro há uma deixa, um estímulo que faz com que o cérebro entre em modo automático e indica qual hábito deve ser adotado. Depois há a rotina, que pode ser física, mental ou emocional. Por fim, há uma recompensa, que auxilia o cérebro a memorizar o loop específico para futuras repetições das ações associadas a rotina.
De acordo com Wendel (2014), existem dois grandes grupos de comportamentos que retratam o que as pessoas querem fazer, mas não conseguem realizar sozinhas. Esses dois grupos de comportamento, e os produtos que possibilitam a prática desses comportamentos, são:

1. Comportamentos que os usuários querem mudar na sua vida diária;

2. Comportamentos inseridos nos produtos, que fazem parte do uso.

Alguns exemplos de comportamentos que as pessoas querem mudar em sua vida diária são: o controle da diabetes, a quitação de débitos de cartão de crédito, a prática de atividades físicas e o envolvimento em comunidades. Já os comportamentos que envolvem o uso dos produtos são todas as ações que o usuário passa a conseguir realizar graças a utilização do produto (ou sistema).

Eyal (2014) criou o modelo gancho para estruturar a construção da formação de hábitos nos usuários por meio da utilização de produtos.

Figura 3 - Modelo gancho (The hook model).

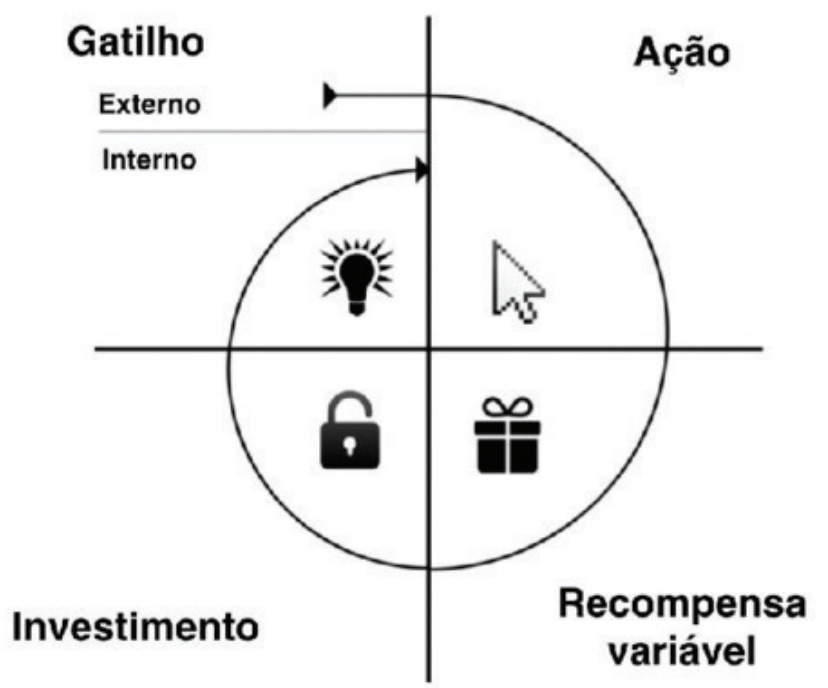

Fonte: Eyal, 2014, p. 4

No modelo gancho o gatilho, interno ou externo, configura-se como o estímulo para a prática de determinada ação. A ação, comportamento executado pelo usuário, serve como uma antecipação da recompensa. A recompensa é qualquer retorno que o produto fornece ao usuário em resposta a pratica de uma ação. Por fim, o investimento é formado pelas ações de suporte que farão com que o usuário tenha engajamento com o produto, garantido, consequentemente, a formação de hábitos. 


\subsection{Projeto da Mudança de Comportamento}

Projetar para a mudança de comportamento consiste em construir produtos que tanto possuem bom design quanto são comportamentalmente efetivos, ou seja, produtos que ajudam as pessoas a mudar o seu comportamento. $\mathrm{O}$ objetivo dessa abordagem é auxiliar as pessoas a fazer coisas que elas querem fazer, mas que ainda não tenham conseguido fazer (WENDEL, 2014).

Para Lockton et. al. (2013) o DfSB objetiva influenciar o comportamento do usuário, por meio do design, para atingir benefícios sociais e ambientais. Já Books (2012) considera que o DfSB envolve a compreensão do comportamento do usuário, a definição de um comportamento alvo, que deverá ser modificado, e a aplicação de estratégias para a promoção da prática de comportamentos sustentáveis.

Lilley (2009) apresenta a estruturação de estratégias para projetar comportamentos sustentáveis:
Os projetistas, quando projetam a tomada de decisão, devem embasar suas escolhas na análise dos comportamentos que podem ser alterados e ajustados. Nessa abordagem diversos fatores devem ser considerados, tais como: características do usuário, tarefas, objetivos e carga cognitiva durante o uso (PETTERSEN e BOKS, 2008). A desconsideração de uma dessas variáveis pode ocasionar o não cumprimento das ações planejadas pelo designer no momento em que o usuário interage com o artefato.

Lockton et. al. (2010) desenvolveram o Método de Design com Intenção - Design with Intent Method (Dwl) - para auxiliar designers na geração de conceitos que influenciam a adoção de comportamentos que conduzam a melhoria de desempenhos e a redução de erros do usuário. A aplicação do Método Dwl começa com o briefing de design que definirá com precisão o comportamento do usuário. $O$ briefing se divide em duas partes: o modo de percepção e o modo de inspiração. No modo de per-

Figura 4 - Estratégias para projetar o comportamento sustentável.

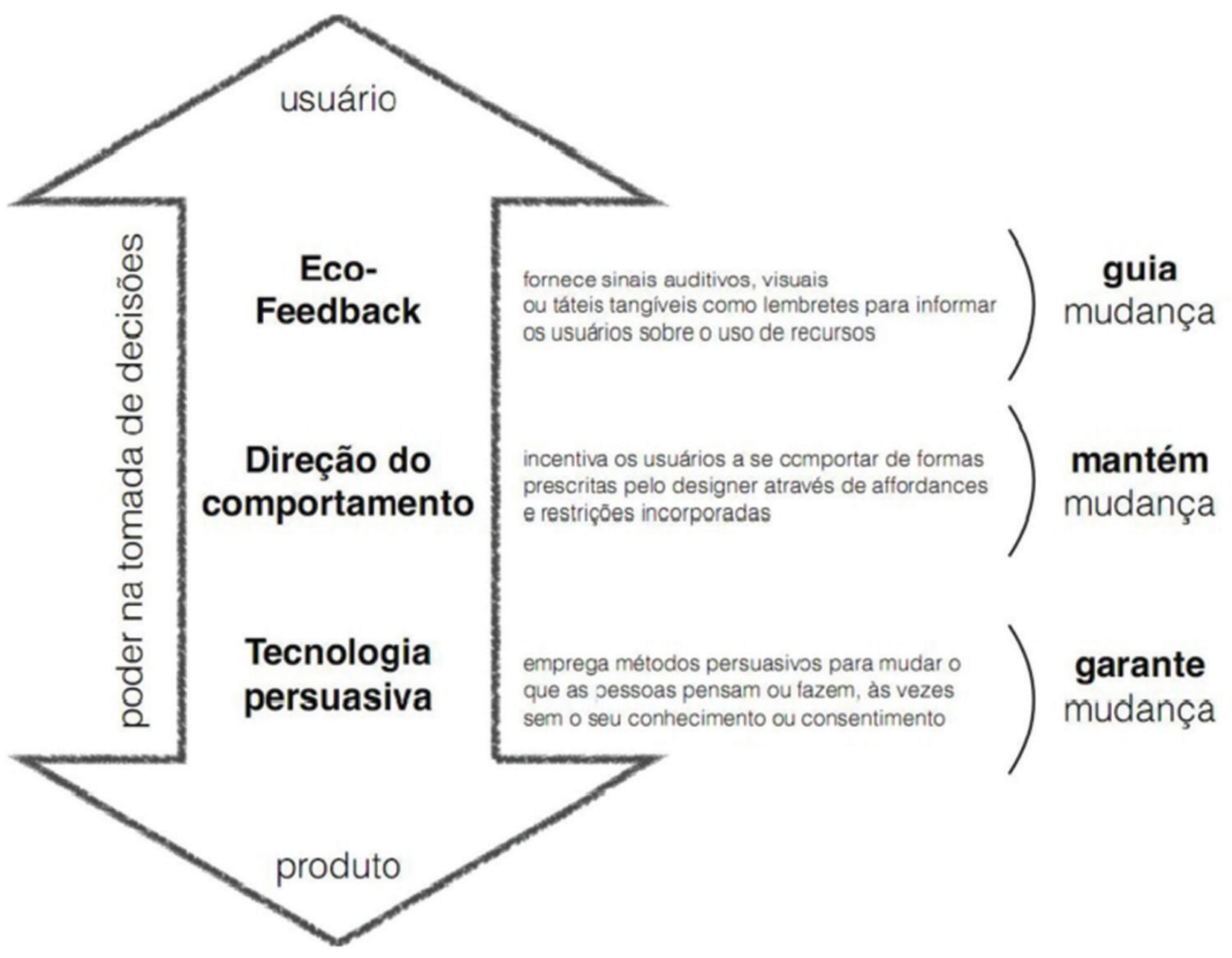


cepção são definidos os comportamentos alvo, que são as ações que se pretende que o usuário execute, através da avaliação das interações entre o usuário e o sistema e das interações entre usuário e usuário. O modo de inspiração possui diversos exemplos que ilustram padrões que podem ser adotados para direcionar o comportamento alvo. Há pelo menos dois padrões para cada uma das seis lentes, que expressam focos, são elas: arquitetural, à prova de erros, persuasiva, visual, cognitiva e segurança. Então, os padrões e exemplos das lentes servirão de inspiração para o desenvolvimento de soluções que atendam ao briefing (LOCKTON et. al., 2010).
O processo de Design para a mudança de comportamento (Designing for Behavior Change) foi sistematizado por Wendel (2014). O referido autor estrutura o processo em quatro etapas, a saber: 1) entender, que envolve a compreensão de como os usuários tomam decisões, para criar um funil de ações e selecionar as estratégias para mudança de comportamento; 2) descobrir, momento em que são elaborados os objetivos do comportamento, que devem estar alinhados com as características dos usuários e com as ações que se pretende promover; 3) Projeto, no qual é definido o plano comportamental, de onde são extraídas histórias dos usuários, para ser definido o design da

Figura 5 - Método de Design com Intenção - Design with Intent Method (Dwl).

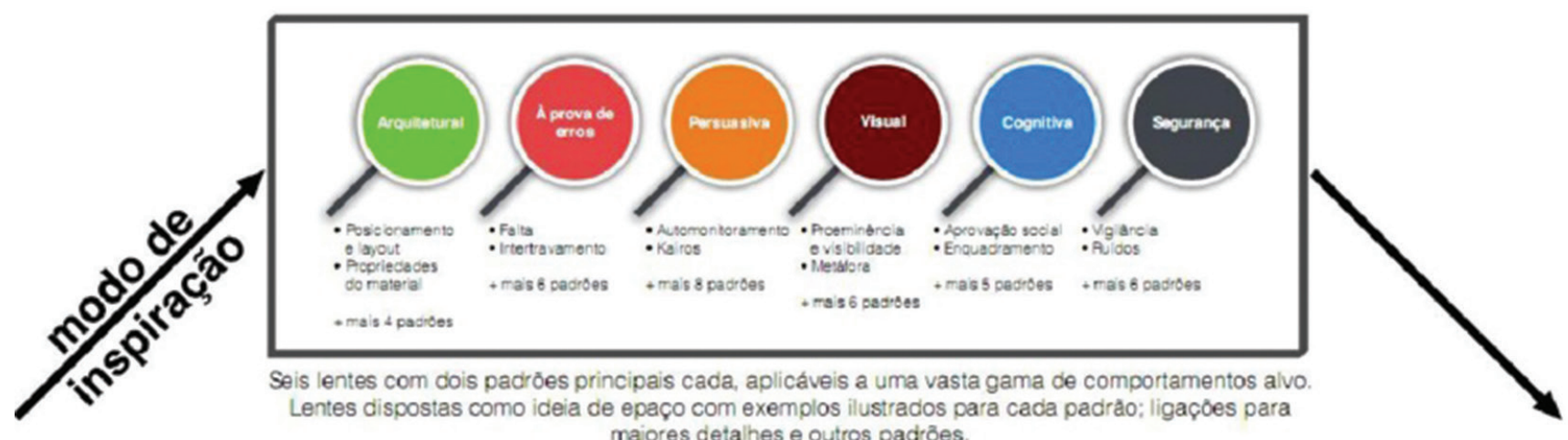
maiores detalhes e outros padröes.

Brief do design do sistema que envolve influenciar o comportamento do usuário

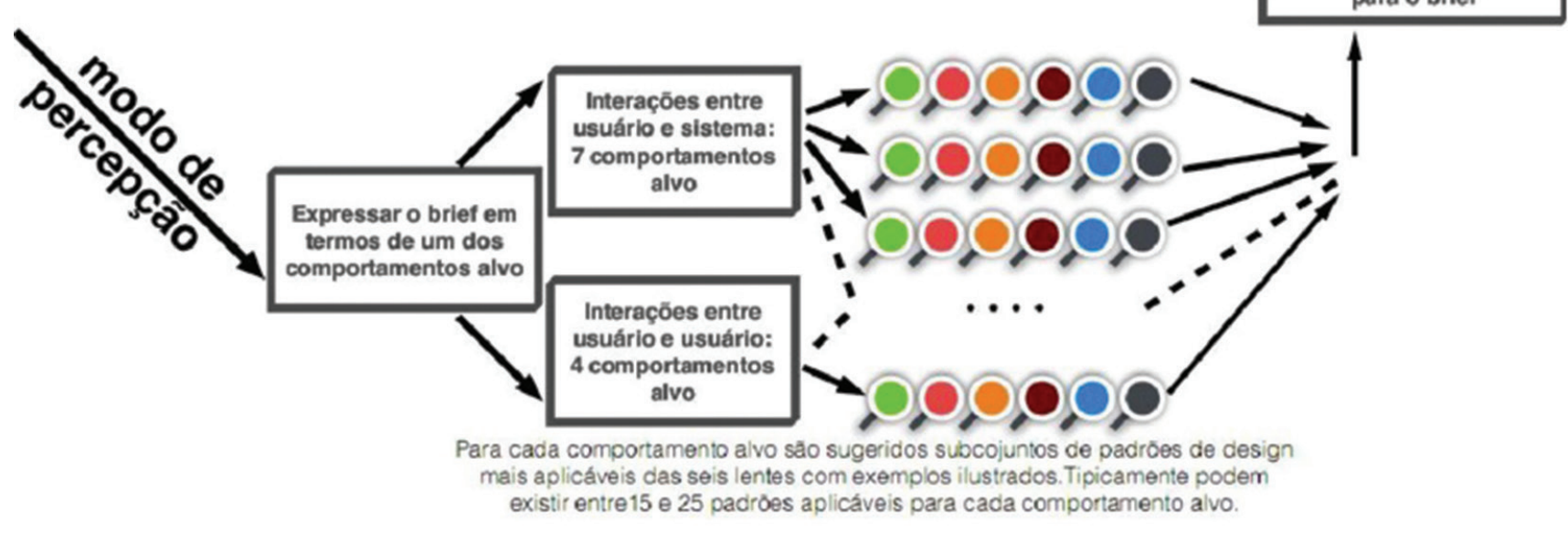

Fonte: Adaptado de Lockton et. al., 2010.

Zachrisson eBoks (2010) propõem um modelo que considera a atitude do usuário perante o comportamento específico determinado pelo design do artefato. Essa abordagem parte do pressuposto que determinada sugestão de comportamento pode gerar diferentes atitudes, portanto, esse modelo pode ser utilizado para determinara melhor estratégia para a mudança de comportamento. interface e do produto; e 4) refinar, onde são construídos protótipos funcionais para serem coletados dados qualitativos e quantitativos sobre o comportamento dos usuários para avaliações de impacto. Nesta etapa poderão ser realizados ajustes com intuito de melhor direcionar os comportamentos desejados e refinar a proposta de design. 
Figura 6 - Relação de atitude do usuário e divisão de controle.

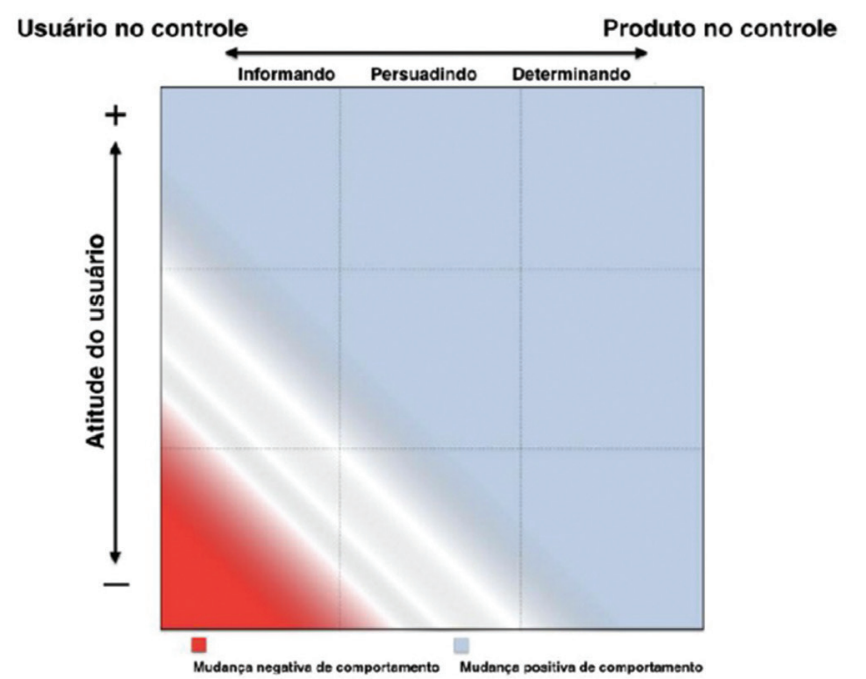

Fonte: Adaptado de Zachrisson e Boks, 2010, p. 13.

Existem diversas estratégias para a mudança de comportamento que podem ser aplicadas em diferentes etapas do processo de design. O contexto de cada projeto definirá qual é a estratégia mais adequada para cada situação e para cada comportamento que se pretende modificar.

\subsection{Produtos que Alteram Comportamentos}

Um exemplo de produto que proporciona a alteração de comportamentos que os usuários querem mudar na sua vida diária é o termostado na empresa Nest. O produto possui um sistema que registra as temperaturas habituais dos usuários para automaticamente fornecê-las nos momentos apropriados. Isso proporciona redução do uso de energia e faz com que os consumidores tenham economia financeira ao mesmo tempo em que contribuem para o meio ambiente.

Figura 8 - Termostato da Nest.

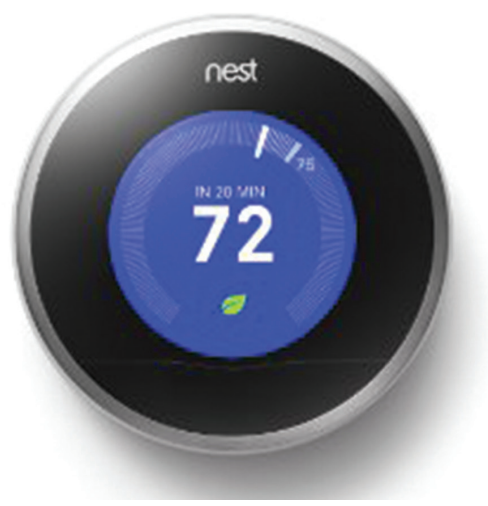

Fonte: Nest, 2014.
Figura 7 - Processo de Design para a mudança de comportamento.

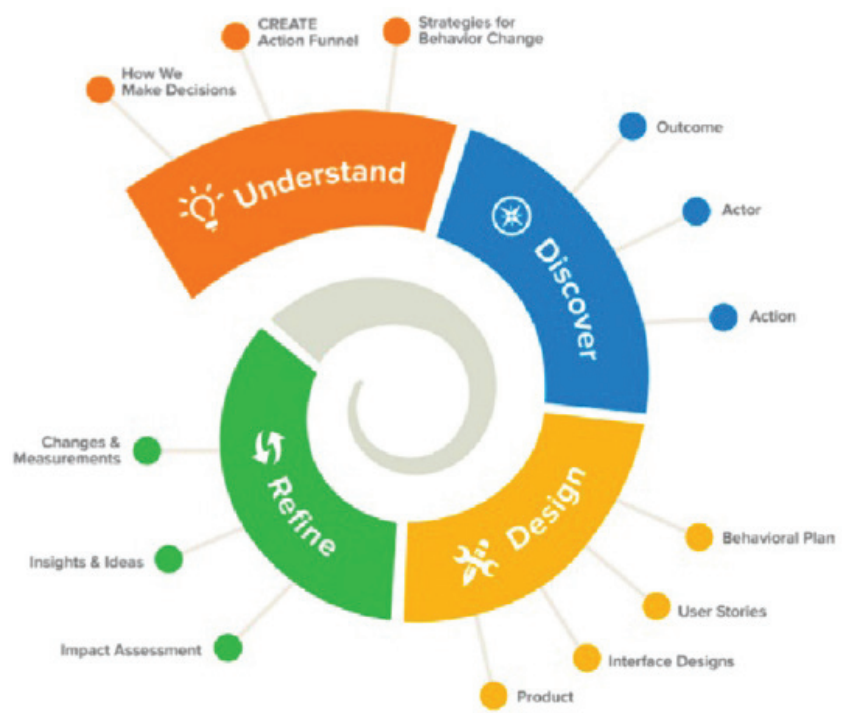

Fonte: Wendel, 2014, p. 72.

Um exemplo de proposta de produto que pode promover a mudança de comportamento por meio do uso é o projeto das novas cédulas de real. As cédulas possuem tamanhos diferentes, de acordo com o valor monetário que possuem. Quanto mais vale a cédula maior o seu tamanho. Isso faz com que as cédulas menores sejam mais utilizadas, por que cabem nas carteiras dos usuários, e as cédulas menores tenham menos circulação, porque não cabem na carteira.

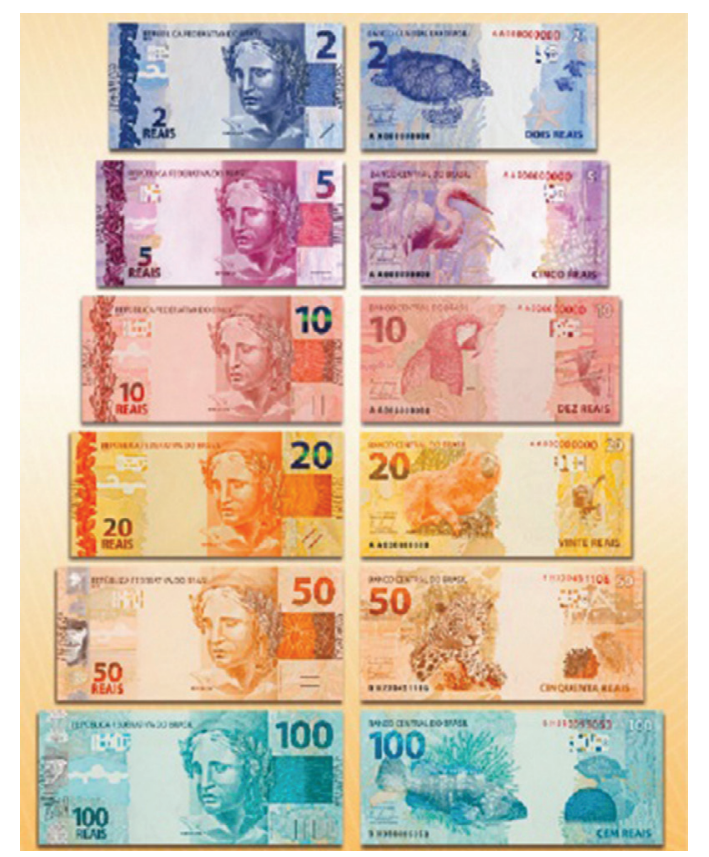

Fonte: Banco Central do Brasil, 2014. 
Os projetos dos dois exemplos apresentados acima se utilizam dos elementos essenciais do Design para a mudança de comportamento propostos por Fogg (2009), são eles: 1) selecionar o comportamento alvo correto; 2)tornar o comportamento alvo fácil de ser realizado; 3) garantir um gatilho que solicita o comportamento.

A Nest definiu o comportamento alvo do termostato como a otimização do gerenciamento da temperatura do ambiente por perceber que essa era uma necessidade dos usuários. Ao utilizar o termostado, a economia de energia e redução da conta de luz servem como gatilhos para o gerenciamento da temperatura do ambiente, que se torna fácil de ser realizado porque é automático (NEST, 2014). No caso das cédulas de real, o Banco Central do Brasil precisava estimular a circulação de notas de menor valor. Então este é o comportamento alvo. Desse modo, o gatilho para a prática do comportamento alvo é a redução do tamanho das cédulas de menor valor e aumento do tamanho das cédulas de maior valor. Assim, fica mais fácil para o consumidor utilizar as cédulas de menor valor.

\subsection{Lacunas na Pesquisa em Design para o Comportamento Sustentável}

Lilley (2009) apresentou a hipótese de que, no projeto de um artefato com estratégias de DfSB, uma série de intervenções provocam mudanças nas soluções de design. Essas passam de "passivas" (ou "informativas") para "assertivas" (ou "persuasivas"), para depois se tornarem "agressivas" (ou "coercitivas"). Contudo, para que isso aconteça as estratégias devem ser executadas de forma sequencial, em resposta a três variáveis:

1. Nível de complacência do usuário;

2. A gravidade das consequências das ações tomadas pelo usuário;

3. O contexto em que a interação entre usuário e artefato ocorre.

Para a referida autora, onde deve ser posicionada cada uma dessas abordagens ("passivas" ou "informativas", "assertiva" ou "persuasivas" e "agressivas" ou "coercitivas") no processo de DfSB ainda é uma questão aberta que necessita discussão. Para classificar com mais precisão as intervenções nessa escala são necessárias mais pesquisas de coleta das percepções dos usuários para medir seus níveis de tolerância a mudanças. Além disso, futuras pesquisas precisam investigar quando a execução automática das ações é aceitável e quando a liberdade de escolha pela prática de uma ação deve ser possibilitada.

Outra questão que permanece aberta diz respeito ao efeito de longo prazo das intervenções. Diversos estudos sobre a efetividade das intervenções estudam apenas os efeitos de curto prazo. Há uma clara necessidade da execução de estudos dos comportamentos duradouros, já que os poucos trabalhos sobre os comportamentos duradouros que foram publicados até o momento não são muito otimistas sobre os efeitos de longo prazo (WEVER, 2012: p. 4).

Para avançar no campo, existem três desafios de pesquisa que precisam ser alcançados: 1) estabelecer uma terminologia comum; 2) estabelecimento de protocolos de pesquisa mais formalizados de pesquisa centrada no usuário para fornecer mais assertivamente as soluções de design; 3) estabelecimento de protocolos de pesquisa mais formalizados para a seleção de comportamentos alvo (BOKS, 2012).

Como o campo de pesquisa em DfSB amadurece, novas questões de pesquisa podem surgir. Uma dessas questões se destina ao contexto dos negócios. Como o DfSB se ajusta ao processo de inovação existente em custos, tempo de consumo e tipos de atividades? (WEVER, 2012). Se existem produtos principalmente para possibilitar a prática de ações, então eles não devem ser projetados para simplesmente consumir menos recursos, os produtos sustentáveis inovadores devem ser projetados para permitir práticas sustentáveis inovadoras (SCOTT et. al., 2011).

\section{CONCLUSÃO}

O presente artigo procurou discutir as abordagens de pesquisa em Design para o comportamento sustentável - Design for Sustainable Behavior (DfSB) - de modo a ampliar o debate acerca do tema e identificar lacunas para futuras pesquisas. Esse trabalho é parte da fundamentação teórica da tese de doutorado do autor.

Ainda são necessárias novas investigações no sentido de se aprofundar no tema e se estabelecer novas discussões. A pesquisa em DfSB tem aumentado significativamente na última década e precisa ser estruturada em uma linha do tempo para que seja pontuada a sua evolução. A partir dessa primeira reflexão, contudo, podem ser colocadas algumas considerações.

O DfSB objetiva auxiliar o usuário a realizar um comportamento que ele está disposto a fazer e não procura persuadir o usuário a praticar ações que ele não gostaria de fazer. Desse modo, a mudança de comportamento acontece no sentido de possibilitar a execução de um comportamento almejado pelo usuário e possibilitado por um artefato. 
A investigação científica em DfSB vem crescendo na última década e tem resultado na estruturação de diversas estratégias que podem ser utilizadas para influenciar a mudança de comportamento do usuário. Como essas estratégias foram desenvolvidas para aplicação em produtos e suas eficácias foram validadas cientificamente, elas passaram a ser utilizadas por projetistas em projetos de produtos que objetivam influenciar a mudança de comportamento dos usuários.

Com isso, as empresas passaram a usufruir da efetividade das estratégias de DfSB modificando o comportamento dos seus consumidores. Uma questão que pode gerar futuras pesquisas está relacionada a mensuração e análise das estratégias de DfSB após o desenvolvimento do projeto de design. As etapas de mensuração e análise, atualmente, acontecem na fase de refinamento da proposta de design. Porém, ainda precisam ser definidas quais são as ações de suporte que devem ser desenvolvidas, e quando elas devem ser aplicadas, para a manutenção de comportamentos de longo prazo depois que o produto está sendo utilizado pelo usuário.

\section{REFERÊNCIAS}

1. Banco Central do Brasil. Segunda família do real. Disponível em: http://www.bcb.gov.br/novasnotas/index.html Acesso em: 05/05/2014.

2. Bhamra, T, Lilley, D, Tang, T (2011) Design for Sustainable Behaviour: Using products to change consumer behaviour, Design Journal, 14(4), pp.427-445, ISSN: 1460-6925.

3. Boks, Casper (2012) Design for Sustainable Behaviour Research Challenges. In: Design for Innovative Value Towards a Sustainable Society, 2012, pp 328-333.

4. Daros, Carolina. Design para a sustentabilidade: oportunidades de inovação a partir dos hábitos de consumo na habitação de interesse social. Dissertação (mestrado) - Universidade Federal do Parana, Setor de Ciências Humanas, Letras e Artes, Programa de Pós-Graduação em Design. Defesa: Curitiba, 19/02/2013.

5. Duhigg, Charles. O poder do hábito: por que fazemos o que fazemos na vida e nos negócios. Tradução: Rafael Mantovani. Rio de Janeiro: Objetiva, 2012.

6. Elias EWA. Dekoninck EA, and Culley, SJC (2007) 'The potential for domestic energy savings through assessing user behaviour and changes in design' In: EcoDesign2007 - 5th International Symposium on Environmentally Conscious Design and Inverse Manufacturing, December 10-13, 2007 in Tokyo, Japan.

7. Elias EWA. Dekoninck EA, and Culley, SJC (2008) 'Assessing User Behaviour for Changes in the Design of Energy Using Domestic Products', In: IEEE International Symposium on Electronics and the Environment ISEE, May 19-22, San Francisco, California, US.

8. Elias EWA. Dekoninck EA, and Culley, SJC (2008a) 'Prioritisation Methodology for User-Centred Design of Energy Using Domestic Products', In: International Design Conference - Design 2008, May 19-22, Dubrovnik, Croatia.

9. Elias EWA. Dekoninck EA, and Culley, SJC (2009) 'Designing for 'Use Phase' Energy Losses of Domestic Products', In: Proceedings of the Institution of Mechanical Engineers, Vol. 222, Part B, Journal of Engineering Manufacture.

10. E-Wise. Objetivo do projeto. Disponível em: http://projetoewise.blogspot.com.br/p/o-projeto.html Acesso em: 05/05/2014.

11. Fogg, B. J. (2009) A Behavior Model for Persuasive Design. In: Persuasive'09, April 26-29, Claremont, California, USA.

12. Forcato, Marcelo dos Santos. Design para o comportamento sustentável: estudo da aplicação do eco-feedback na interface de lavadora de roupas. Dissertação (mestrado) - Universidade Federal do Parana, Setor de Ciências Humanas, Letras e Artes, Programa de Pós-Graduação em Design. Defesa: Curitiba, 18/02/2014.

13. Froehlich, Jon (2009) Sensing and Feedback of Everyday Activities to Promote Environmentally Sustainable Behaviors. In: UbiComp 2009, Sep 30 - Oct 3, 2009, Orlando, Florida, USA.

14. Geelen, D., Keyson, D., Boess, S. and Brezet, H. (2012) 'Exploring the use of a game to stimulate energy saving in households', J. Design Research, Vol. 10, Nos. 1/2, pp.102-120.

15. Haines, V., Mitchell, V. and Mallaband, B. (2012) 'Merging a practice-orientated approach with an engineerin 
1. g-driven product development: a case study on home improvement', J. Design Research, Vol. 10, Nos. 1/2, pp.2849.

2. Jelsma, J. and Knot, M. (2002) Designing environmentally efficient services; a 'script' approach, The Journal of Sustainable Product Design, Vol. 2, pp 119-130.

3. Kahneman, Daniel. Rápido e Devagar: Duas Formas de Pensar. Tradução Cássio de Arantes Leite. Rio de Janeiro: Objetiva, 2012.

4. Kuijer, L. and de Jong, A. (2012) 'Identifying design opportunities for reduced household resource consumption: exploring practices of thermal comfort', J. Design Research, Vol. 10, Nos. 1/2, pp.67-85.

5. Laitala, K. and Boks, C. (2012) 'Sustainable clothing design: use matters', J. Design Research, Vol. 10, Nos. 1/2, pp.121-139.

6. Lilley, D. (2009) Design for sustainable behaviour: strategies and perceptions. In: Design Studies, Volume 30, Issue 6, November 2009, Pages 704-720.

7. Lockton, D., Harrison, D. J., Cain, R., Stanton, N. A., \& Jennings, P. (2013). Exploring problem-framing through behavioural heuristics. International Journal of Design, 7(1), 37-53.

8. Lockton, D., Harrison, D.J., Stanton, N.A.. The Design with Intent Method: a design tool for influencing user behaviour. In: Applied Ergonomics. Vol. 41, No.3, pp. 382 392, May 2010.

9. McCalley, L. T. (2006) From motivation and cognition theories to everyday applications and back again: the case of product-integrated information and feedback, Energy Policy, Vol. 34, No. 2, pp. 129-137.

10. McCalley, L. T. and Midden, C. J. (2002) Energy conservation through product-integrated feedback: The roles of goal-setting and social orientation, Journal of Economic Psychology, Vol. 23, pp. 589-603.

11. McCalley, L. T. and Midden, C. J. H. (2006a) Making Energy Feedback Work In: User Behavior and Technology Development: Shaping Sustainable Relations Between Con- sumers and Technologies Verbeek, P.-P. and Slob, A. (eds) Springer, The Netherlands, pp. 127-135.

12. Nest. Termostat. Disponível em: https://nest.com Acesso em: 05/05/2014.

13. Pettersen, I. N.; Boks, C.. The ethics in balancing control and freedom when engineering solutions for sustainable behaviour. In: International Journal of Sustainable Engineering. Vol. 1, Issue 4, pp. 287-297, 2008.

14. Rodriguez, E. (2004) User research and eco-ergonomics: encouraging environmentally effective behaviours in product users through the industrial design process, In: International Conference on Sustainability Engineering and Science, Sheraton Hotel and T owers, Auckland, New Zealand, 6th - 9th July 2004.

15. Rodriguez, E. and Boks, C. (2005) How design of products affects user behaviour and vice versa: the environmental implications, In: Environmentally Conscious Design and Inverse Manufacturing 2005, 12th - 14th December 2005.

16. Scott, Kakee; Bakker, Conny; Quist, Jaco (2012) Designing change by living change. In: Design Studies, Volume 33, Issue 3, May 2012, Pages 279-297.

17. Secomandi, F.. Thinking through the service interface The Philips Direct Life. In: Design Philosophy Papers. Issue Design, change and politics, 1/2013.

18. Skoon. Objetivo do projeto. Disponível em: http://projeto-skoon.blogspot.com.br/p/sobre-o-skoon.html Acesso em: 05/05/2014.

19. Van Dam, S.S., Bakker, C.A. and Van Hal, J.D.M. (2012) 'Insights into the design, use and implementation of home energy management systems', J. Design Research, Vol. 10, Nos. 1/2, pp.86-101.

20. Wendel, Stephen (2014) Designing for Behavior Change: Applying Psychology and Behavioral Economics. O'Reilly, Gravenstein Highway North, Sebastopol, 2014.

21. Wever, R (2011). Design for volume optimization of packaging for durable goods. Packaging Technology and Science, 24(4), 211-222. 
22. Wever, R. (2012). Editorial: Special issue on design for sustainable behaviour. Journal of Design Research, 10(1-2), 1-6.

23. Wever, R., van Kuijk, J. and Boks, C. (2008) User-centred Design for Sustainable Behaviour, International Journal of Sustainable Engineering, Vol. 1, No. 1, pp.9-20.

24. Wever, R; Boks, CB \& Stevels, ALN (2006). Balancing environmental performance with sales functionalities in packaging for consumer electronic products. In JR Duflou, W Dewulf, B Willems \& T Devoldere (Eds.), Proceedings of the 13th CIRP International Conference on Life Cycle Engineering (pp. 323-328).

25.Zachrisson, Johannes; Boks, Casper (2010) When to apply different design for sustainable behaviour strategies? In: Knowledge Collaboration \& Learning for Sustainable Innovation. ERSCP-EMSU conference, Delft, The Netherlands, October 25-29, 2010. 